\title{
Características de las variedades de maíz chococeño (Zea mays), de las comunidades Embera del Atrato medio antioqueño*
}

\section{Chococeño corn crop varities characteristics in indigenous people Embera from middle Atrato Antioquiariver}

\author{
José Ubeimar Arango Arroyave ${ }^{1}$, Gloria PatriciaZuluaga Sánchez ${ }^{2}$, \\ Diana Isabel Peñarete Ramírez ${ }^{3}$
}

\section{RESUMEN}

El objetivo de este artículo es la identificación, descripción y análisis de las caracteristicas del maíz Rea mays), y sus variedades con principal énfasis en la raza Chococeño, en cuanto a líneas genéticas, aspectos germinativos y de vigor, formas de producción, conservación y usos. Para la recolección de la información se usó como estrategia metodológica una combinación de técnicas de investigación cualitativas y cuantitativas, tales como la observación participante, recorridos de campo, talleres y pruebas de laboratorio para determinar nivel de viabilidad y de germinación de las variedades de maiz encontradas en los territorios indigenas objeto de la presente investigación. Dentro de los resultados se destaca la alta diversidad intraespecífica del maiz, informando 6 variedades, las cuales se adaptan a unas condiciones climáticas con altas restricciones para muchos materiales mejorados genéticamente.

Palabras clave: Producción tradicional de maíz; Embera; Atrato medio; Maiz chococeño.

\section{ABSTRACT}

In this theme it will analyze the information about Chococeño corn varieties (Zea mays) in Embera indigenous people of Middle Atrato in town about aspects as: Using, production traditional systems conservation, identification and analysis of some characteristics expressed at the germination potential and of power. This investigation had as methodological strategy several quantitative investigation techniques and qualitative such as: participant observation, field study, and laboratory proof to determinate Chococeño corn viability percent and germination found in the varieties different. The results show the wide intraespecific diversity find 6 corn varieties, they are adapted a different conditions of climate with difficults for improved genetic varieties.
* El presente artículo hace parte del trabajo de investigación «Estrategias de producción, extracción y protección en los territorios de las comunidades Embera de Jarapetó, Jengadóy Ñarangué del MedioAtrato Antioqueño", realizado en coordinación y a solicitud de la Organización Indígena de Antioquia (OIA) en convenio con el Instituto de Investigaciones Ambientales del Pacífico (IIAP).

1. Ingeniero Agrónomo, Asesor Técnico Organización Indígena de Antioquia (OIA).

e-mail: imandau@yahoo.ca

2. Profesora Asociada, Universidad Nacional de Colombia.

e-mail: gpzuluag@unalmed.edu.co

3. Ingeniera Forestal, ex asesora Organización Indígena de Antioquia (OIA).

Recibido: Mayo 14, 2008

Aceptado: Junio 30, 2008

Keywords: Corn production traditional system; Embera; Middle Atrato; Chococeño corn.

\section{ÁREA DE ESTUDIO Y CARACTERÍSTICAS}

La presente investigación se realizó en los territorios Embera de la región del Atrato medio antioqueño, específicamente en las comunidades indígenas de Jarapetó y Jengadó del munici- 


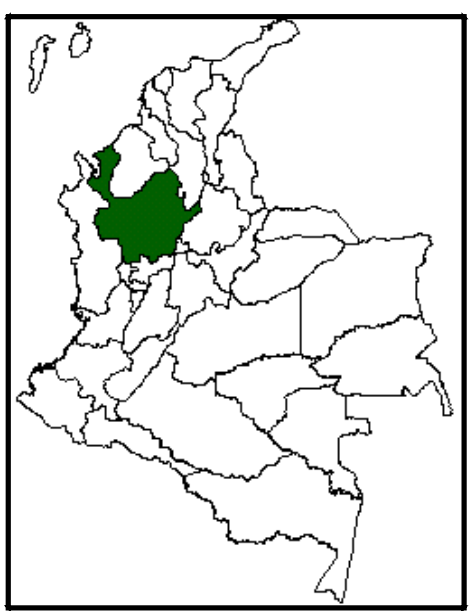

Figura 1. Territorios de comunidades indígenas del Atrato medio antioqueño (área de estudio).

Fuente: OIA. Elaboración Hernán Darío

Posada Garcés, Asesor Programa Territorio y Medio Ambiente

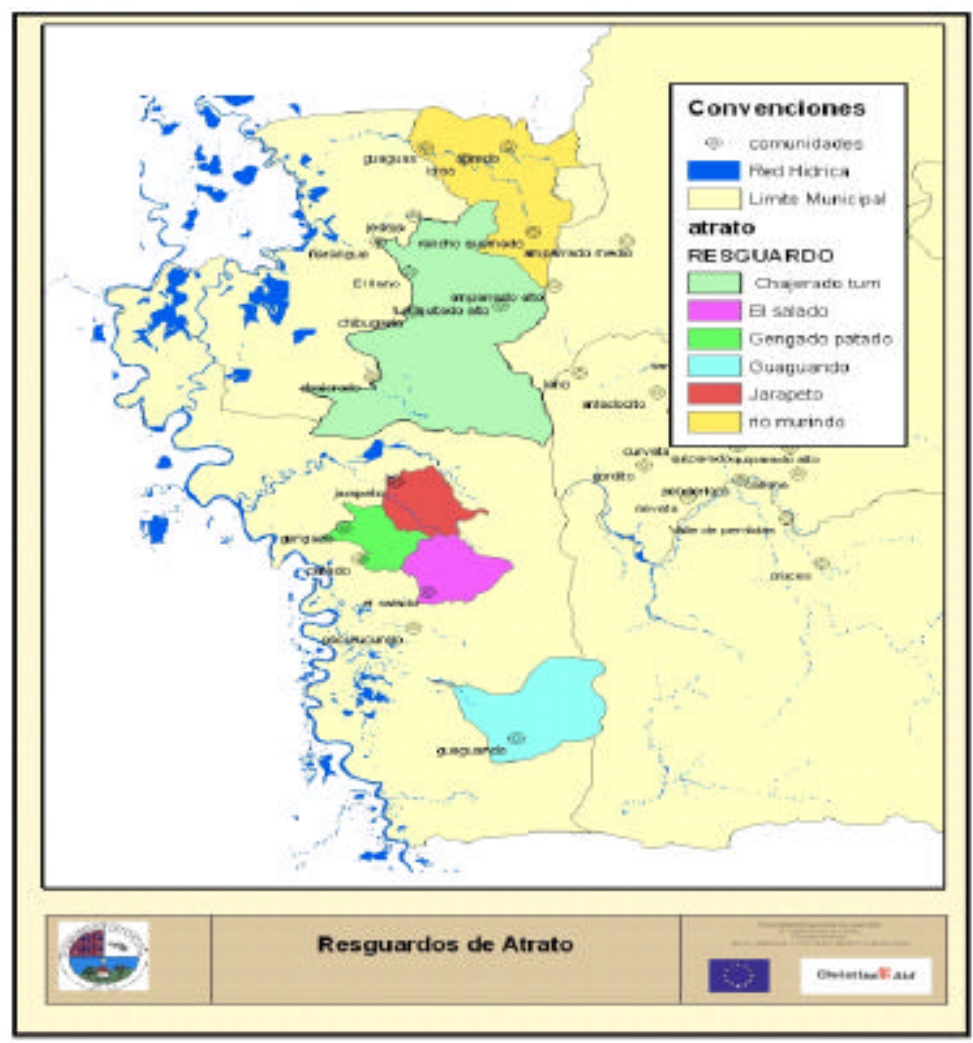

caracteriza por la apertura de claros en el bosque para el establecimiento de cultivos de maíz y plátano principalmente, los cuales son abandonados al cabo de dos o tres años, cuando empieza a disminuir la producción, por la baja fertilidad de los suelos o por la alta presencia de plagas y enfermedades, pasando luego a un proceso de sucesión del bosque a manera de rastrojeras, las cuales después de un período de descanso vuelven a un proceso de rotación o de apertura de nuevos espacios de cultivo (Betancourt y Zuluaga, 1988, Arango y Peñarete, 2000).

El maíz tiene un significado muy importante en la cultura Embera, porque es una de las principales especies vegetales de su dieta después de las musáceas, además por ser aprovechada tradicionalmente en la preparación de la chicha, bebida básica utilizada en las mingas o trabajo colectivo. Su cultivo se realiza generalmente en "monoparcelas", que se distinguen por una alta diversidad intraespecífica o diversidad genética dentro de la misma especie, por ejemplo, se han informado diez variedades para el plátano y seis para el caso del maíz en una misma parcela (Arango y 
Peñarete, 2000; Betancourt y Zuluaga, 1988; Gálvez, 1997; García y Velásquez, 1992). Su cultivo se establece mediante el sistema de roza, tumba y pudre (en otros ecosistemas con menos lluvia anual se realiza el sistema tumba, roza y quema, pero en los territorios del Atrato medio, las condiciones climáticas impiden la quema, la cual es reemplazada por un proceso donde el material vegetal tumbado y rozado se deja podrir, lo cual constituye una estrategia interesante de incorporación de materia orgánica al sistema), el cual es una práctica de uso y manejo del suelo propia de la agricultura migratoria de bosques húmedos tropicales, la cual según el Centro Agronómico Tropical de Investigación y Enseñanza (CATIE) y la Organización para Estudios Tropicales (OTS) (1986), se clasifica dentro de la categoría de sistemas agroforestales secuenciales. Entre la siembra y la cosecha no se ejecutan prácticas de cuidado y mantenimiento. A los tres o tres meses y medio se recoge una parte de la cosecha en chócolo, consumiéndose asado o en dulces. A los cuatro meses se recoge la totalidad de la cosecha principal, cuando el maíz ya está seco y es almacenado en canastos o en una especie de cilindro elaborado con la corteza de balzo; generalmente se guarda en zarzos, dejándolo siempre con el capacho para evitar el ataque de gorgojos y roedores. Con respecto a las semillas, se sabe que las custodian las personas de mayor edad de la comunidad, también es común que se intercambien en visitas a parientes o amigos tras largas jornadas de camino, lo que permite mantener una base genética de alta diversidad (Betancourt y Zuluaga, 1988; Arango y Peñarete, 2000).

En lo que respecta propiamente a las variedades de maíz encontradas, es importante resaltar la presencia del maíz chococeño, chococito o indio, el cual fue informado por (Patiño, 1964, Mesa 1995), como una especie típica del Pacífico colombiano, región de la cual el medio Atrato hace parte. Patiño (1964), afirma que el chococito, es una de las razas del maíz más primitivas entre las conocidas, no sólo por su morfología (plantas retoñadoras; mazorcas pequeñas con granos pequeños, cristalinos, reventadores o harinosos) y hábitos (crece y se desarrolla en zonas húmedas, y en muchos casos se comporta como una planta acuática), además por su sistema de propagación, donde la intervención del hombre es mínima, pues se limita a regar la semilla sobre el rastrojo, cortar éste encima, y abandonar la sementera hasta la formación y cosecha de la mazorca, no existiendo la quema inicial del monte ni la desyerba del cultivo que son indispensables en el sistema clásico de otras variedades de maíz. El cultivo de maíz en las comunidades Embera está altamente integrado en el ecosistema dónde se desarrolla; muestra de ello es la gran cantidad de otras hierbas con las que convive y se desarrolla en las áreas de parcela.

Es de resaltar que las variedades informadas en este trabajo tienden a retoñar inclusive en condiciones de inundación, si se considera las condiciones de alta humedad del Atrato medio (más de 5.000 mm de lámina de agua al año), que son limitantes para muchas plantas "mejoradas", que no se desarrollan bien o presentan muchos problemas fitosanitarios. La zona de dispersión de esta variedad fue estudiada y registrada por Patiño (1964), quien consideró un área de unos $100.000 \mathrm{~km}^{2}$, coincidiendo con la región conocida como Chocó Biogeográfico, que incluye territorios en Colombia, Ecuador y Panamá. En el caso de Colombia se tienen unos $80.000 \mathrm{~km}^{2}$, de los cuales la mitad pertenecen al Chocó y el resto a Antioquia, Valle del Cauca, Caldas, Cauca y Nariño. En el Ecuador se encontró la variedad en toda la provincia de Esmeraldas y una porción de la de Pichincha, con un total que se calcula en unos $18.000 \mathrm{~km}^{2}$; en Panamá, no se tiene datos del área, pero se sabe que es mucho más reducida y dispersa.

\section{METODOLOGÍA}

El presente trabajo se llevó a cabo en cuatro fases:

Fase I . Recorridos en los territorios deJarapetó, Jengadó y Ñarangué (Vigía del Fuerte y Murindó), con énfasis en observación directa, observación participante y entrevistas semiestructuradas, con el fin de indagar sobre el conocimiento tradicional de los indígenas sobre los agroecosistemas de maíz de bosque húmedo y muy húmedo, así como de algunos aspectos etnobotánicos.

Fase II. Colecta, reconocimiento e identificación de las variedades encontradas, previo consentimiento y aprobación de las comunidades Indígenas.

Fase III. Dos visitas al banco de germoplasma de maíz del centro CORPOICA Tulio Ospina de Bello para la identificación, reconocimiento y 
Bioetnia Volumen 5 № 1 (enero-junio), 2008

Tabla 1

Descripción de tipos de maíz raza chococeño encontradas comunidades del Atrato medio antioqueño

\begin{tabular}{lcccc}
\hline Tipo de maíz & Diámetro $(\mathbf{c m})$ & Altura $(\mathbf{c m})$ & No hileras & Observaciones \\
\hline Negro & 3.5 & 11.3 & 16 & Hileras onduladas tercio inferior \\
\hline Rojo & 3.3 & 8.5 & 18 & Hileras onduladas tercio inferior \\
\hline Café & 4.2 & 11 & 22 & Hileras ligeramente onduladas desde el \\
& & & 17 & tercio inferior hasta el superior \\
\hline Amarillo & 3.7 & 10.2 & 18 & Hileras onduladas tercio inferior \\
\hline Blanco & 3.7 & 10.6 & 18 & Hileras ligeramente onduladas desde la \\
\hline Anaranjado & 3.6 & 14.1 & & base hasta la parte superior \\
\hline
\end{tabular}

comparación de las colecciones de la raza de maíz chococeño presentes y colectadas en Colombia con las informadas en este trabajo.

Fase IV. Descripción de las variedades con énfasis en designación de la variedad, diámetro promedio de la mazorca, altura, número de hileras y observaciones. Pruebas de germinación y viabilidad (prueba del tetrazolio) a tres y nueve meses después de cosechado, en el Laboratorio de Fisiología Vegetal de la Universidad Nacional de Colombia sede Medellín.

\section{RESULTADOS}

Las líneas de maíz reconocidas y colectadas corresponden a los nombres de maíz amarillo (be kuara) (los nombres que aparecen en paréntesis corresponden con sus respectivas denominaciones en lengua Embera), blanco (be torró), negro (be paima), rojo (be purru), café (nejarrabe), naranjado (vitoto). Genéricamente los Embera del medio atrato lo llaman pajarito, mientras los del occidente antioqueño y Urabá lo designan con el nombre de chumilo e indio. Estas muestras se llevaron al banco de germoplasma de maíz de CORPOICA "Tulio Ospina", ubicado en el municipio de Bello, donde se pudo establecer que corresponden a la raza colombiana de chococeño llamado también con el nombre de chococito, que están dentro de las colecciones de maíz colectadas en Colombia desde 1957. A continuación se presentan en las Tablas 1 y 2 algunas características de los tipos de maíz reconocidos y encontrados en territorios de comunidades Embera del Atrato medio antioqueño, donde se resaltan además sus respectivos usos.

Ensayos de germinación y de viabilidad. Las pruebas de germinación y de viabilidad realizadas a nivel de laboratorio contemplaron un intervalo de almacenamiento entre 3 meses y 9 meses, tiempo este último en el cual suele almacenarse el maíz por parte de estas comunidades, porque sólo se siembra una sola vez al añoy no se hace de manera intensiva porque todas las labores de pre y establecimiento de este agroecosistema se basa en períodos de descanso, recuperación y rotación de barbechos, esto hace que sea importante conocer en este tiempo el potencial de vigor y la calidad germinativa de los diferentes fenotipos de maíz, por la importancia que presenta este cereal en la dieta y sistema alimentario de estas comunidades, así como en la capacidad adaptativa a las condiciones ambientales de alta humedad y de suelos ácidos. Los ensayos realizados con cada una de las variedades de maíz colectadas e identificadas a nivel de prueba de germinación y de viabilidad arrojaron los resultados que se exponen en las Tablas 3 a 6.

\section{DISCUSIÓN}

En los valores informados en las Tablas 3 y 5 se aprecia la existencia de diferencias en los porcentajes de germinación, mientras que para las semillas de tres meses se obtuvo un promedio de 97\% de germinación contados a partir del cuarto día; para los de doce meses fue de $64 \%$, siendo más retardada, contados a partir del noveno día, notándose además la disminución del porcentaje de germinación por variedad. Un caso muy mar- 
Tabla 2

Algunas características de los diferentes tipos de maíz identificadas en los sistemas de producción Embera del Atrato medio antioqueño (A)

\begin{tabular}{|c|c|c|c|}
\hline Línea genética & Cómo se reconoce & Época de siembra & Tiempo de cosecha \\
\hline Vitoto anaranjado & Mazorca de color anaranjado fuerte & $\begin{array}{l}\text { Del } 10 \text { al } 15 \text { de marzo; del } 20 \text { al } \\
25 \text { de abril, del } 10 \text { al } 20 \text { de junio }\end{array}$ & Cuatro meses \\
\hline Maíz negro & $\begin{array}{l}\text { Mazorca morada oscura, el tallo de la } \\
\text { planta es más delgado que el de las } \\
\text { variedades anteriores }\end{array}$ & $\begin{array}{l}\text { Del } 10 \text { al } 15 \text { de marzo; del } 20 \text { al } \\
25 \text { de abril, del } 10 \text { al } 20 \text { de junio }\end{array}$ & Cuatro meses \\
\hline Maíz blanco & Sus granos son de color blanco & $\begin{array}{l}\text { Del } 10 \text { al } 15 \text { de marzo; del } 20 \text { al } \\
25 \text { de abril, del } 10 \text { al } 20 \text { de junio }\end{array}$ & Cuatro meses \\
\hline $\begin{array}{l}\text { Maíz café } \\
\text { nejarrabé }\end{array}$ & Sus granos son de color café oscuro & Marzo, abril, mayo & Cuatro meses \\
\hline Maíz rojo & $\begin{array}{l}\text { Mazorcas de color rojo intenso, } \\
\text { gruesas, el tallo de la planta es } \\
\text { delgado y la mazorca es de porte bajo }\end{array}$ & $\begin{array}{l}\text { Del } 10 \text { al } 15 \text { de marzo; del } 20 \text { al } \\
25 \text { de abril, del } 10 \text { al } 20 \text { de junio }\end{array}$ & Cuatro meses \\
\hline Chumilo amarillo & $\begin{array}{l}\text { Es muy parecido al anterior, pero la } \\
\text { mazorca es más delgada y larga, sus } \\
\text { granos son de un color amarillo más } \\
\text { fuerte }\end{array}$ & $\begin{array}{l}\text { Del } 10 \text { al } 15 \text { de marzo; del } 20 \text { al } \\
25 \text { de abril, del } 10 \text { al } 20 \text { de junio }\end{array}$ & Cuatro meses \\
\hline
\end{tabular}

Tabla 2

Algunas características de los diferentes tipos de maíz identificadas en los sistemas de producción Embera del Atrato medio antioqueño (B)

\begin{tabular}{|c|c|c|c|}
\hline Línea genética & $\begin{array}{c}\text { Problemas } \\
\text { fitosanitarios }\end{array}$ & $\begin{array}{l}\text { Producción } \\
\text { en almud }\end{array}$ & Usos \\
\hline Vitoto anaranjado & $\begin{array}{l}\text { Michitá, ratón, guagua, } \\
\text { tatabro }\end{array}$ & $\begin{array}{l}\text { Se siembra } 1 \text { almud y se } \\
\text { recoge de } 13 \text { a } 14 \text { almud }\end{array}$ & $\begin{array}{l}\text { Se prepara chicha, colada; } \\
\text { cuando se encuentra en estado } \\
\text { de chócolo, arepa, mazamorra, } \\
\text { envuelto. }\end{array}$ \\
\hline Maíz negro & $\begin{array}{l}\text { Michitá, ratón, guagua, } \\
\text { tatabro }\end{array}$ & $\begin{array}{l}\text { Se siembra } 1 \text { almud y se } \\
\text { recoge de ( } 13 \text { a } 14 \text { almud }\end{array}$ & $\begin{array}{l}\text { Se prepara chicha, colada; } \\
\text { cuando se encuentra en estado } \\
\text { de chócolo, arepa, mazamorra, } \\
\text { envuelto. }\end{array}$ \\
\hline Maíz blanco & $\begin{array}{l}\text { Michitá, ratón, guagua, } \\
\text { tatabro }\end{array}$ & $\begin{array}{l}\text { Se siembra } 1 \text { almud y se } \\
\text { recoge de } 13 \text { a } 14 \text { almud }\end{array}$ & $\begin{array}{l}\text { Se prepara chicha, colada; } \\
\text { cuando se encuentra en estado } \\
\text { de chócolo, arepa, mazamorra, } \\
\text { envuelto. }\end{array}$ \\
\hline $\begin{array}{l}\text { Maíz café } \\
\text { nejarrabé }\end{array}$ & $\begin{array}{l}\text { Arriera, Michitá, ratón, } \\
\text { guagua, tatabro }\end{array}$ & $\begin{array}{l}\text { Se siembra } 1 \text { almud, se } \\
\text { recoge } 15\end{array}$ & $\begin{array}{l}\text { Se prepara chicha, colada; } \\
\text { cuando se encuentra en estado } \\
\text { de chócolo, arepa, mazamorra, } \\
\text { envuelto. }\end{array}$ \\
\hline Maíz rojo & Michitá & $\begin{array}{l}\text { Se siembra } 1 \text { almud y se } \\
\text { recoge de } 1314 \text { almud }\end{array}$ & $\begin{array}{l}\text { Se prepara chicha, colada; } \\
\text { cuando se encuentra en estado } \\
\text { de chócolo, arepa, mazamorra, } \\
\text { envuelto. }\end{array}$ \\
\hline Chumilo amarillo & Gorgojo & $\begin{array}{l}\text { Se siembraun } 1 \text { almud y se } \\
\text { recoge de } 13 \text { a } 14 \text { almud }\end{array}$ & $\begin{array}{l}\text { Se prepara chicha, colada; } \\
\text { cuando se encuentra en estado } \\
\text { de chócolo, arepa, mazamorra, } \\
\text { envuelto (para autoconsumo) y } \\
\text { se vende. }\end{array}$ \\
\hline
\end{tabular}

cado fue para la variedad de maíz rojo que de $96 \%$ a tres meses pasó a $12 \%$ en el período de un año. Las causas que determinan las diferencias en los porcentajes de germinación pueden obedecer a varios factores entre los que se destacan la madurez fisiológica, viabilidad, condiciones y período de almacenamiento, sanidad, tratamientos con bio-estimulantes, inhibidores que se encuen- 
Bioetnia Volumen 5 № 1 (enero-junio), 2008

Tabla 3

Pruebas de germinación en maíz a 3 meses

\begin{tabular}{|lccccc}
\hline Tipo de maíz & No de semillas & Días de germinación & Germinaron & No germinaron $\%$ germinación \\
\hline Blanco & 50 & 4 & 44 & 6 & 94 \\
\hline Negro & 50 & 4 & 47 & 3 & 98 \\
\hline Café & 50 & 4 & 49 & 1 & 99 \\
\hline Rojo & 50 & 4 & 45 & 5 & 96 \\
\hline Naranjado & 50 & 4 & 47 & 3 & 97 \\
\hline Amarillo & 50 & 4 & 48 & 2 & 98 \\
\hline
\end{tabular}

Tabla 4

Pruebas de viabilidad en maíz a 3 meses

\begin{tabular}{lccccc}
\hline Tipo de maíz & No de semillas & Tiñó & No tiñó & \% viabilidad & Rango de coloración \\
\hline Blanco & 50 & 48 & 2 & 98 & 1 \\
\hline Negro & 50 & 49 & 1 & 99 & 1 \\
\hline Café & 50 & 47 & 3 & 97 & 1 \\
Rojo & 50 & 49 & 1 & 96 & 1 \\
\hline Naranjado & 50 & 49 & 1 & 99 & 1 \\
\hline Amarillo & 50 & 46 & 4 & 96 & 1 \\
\hline
\end{tabular}

Tabla 5

Pruebas de germinación en maíz a 9 meses

\begin{tabular}{|lccccc}
\hline Tipo de maíz & No de semillas & Días de germinación & Germinaron & No germinaron & \% germinación \\
\hline Blanco & 50 & 9 & 48 & 2 & 98 \\
\hline Negro & 50 & 9 & 31 & 19 & 62 \\
\hline Café & 50 & 9 & 33 & 17 & 66 \\
\hline Rojo & 50 & 9 & 6 & 44 & 12 \\
\hline Naranjado & 50 & 9 & 39 & 11 & 78 \\
\hline Amarillo & 50 & 9 & 34 & 16 & 68 \\
\hline
\end{tabular}

Tabla 6

Pruebas de viabilidad en maíz a 9 meses

\begin{tabular}{|lccccc}
\hline Tipo de maíz & No de semillas & Tiñó & No tiñó & \% viabilidad & Rango de coloración \\
\hline Blanco & 50 & 50 & 0 & 100 & 1 \\
\hline Negro & 50 & 50 & 0 & 100 & 1 \\
\hline Café & 50 & 48 & 2 & 96 & 1 \\
\hline Rojo & 50 & 46 & 4 & 92 & 1 \\
\hline Naranjado & 50 & 50 & 0 & 100 & 1 \\
\hline Amarillo & 50 & 50 & 0 & 100 & 1 \\
\hline
\end{tabular}


tran en la capa externa de las semillas y latencia. Entre los factores externos se deben considerar: temperatura, luz, humedad y atmósfera gaseosa, siendo los resultados más óptimos cuando las primeras y las segundas se complementan en forma favorable (Montoya, 1993). Otro factor que se debe considerar es la contaminación presente en el laboratorio (polvo y polución, generados por la cercanía a la vía regional y por la reestructuración del edificio donde se encuentra el laboratorio).

En otras investigaciones se reportan porcentajes menores de germinación, sin embargo, estos no fueron realizados a nivel de laboratorio. Uno de ellos fue informado por Castro y Cortés (1992) en Tumaco, donde se reportaron porcentajes de germinación en campo para el chococeño de $40 \%$ y 60\%. Por su parte Márquez (1994), en Nariño (municipio Olaya Herrera) informa porcentajes de $90 \%$ en campo para el chococeño blanco. En lo que respecta a la viabilidad, los valores son muy similares entre las variedades descritas en las Tablas 4 y 6 superiores a 90\%. Prácticamente no se presentan diferencias en los porcentajes de viabilidad, lo que indica que son semillas con mucha capacidad para sobrevivir bajo condiciones desfavorables de campo, lo cual es de gran importancia porque en buena medida estas comunidades no tienen muchas técnicas de conservación y almacenaje de las semillas.

El hecho de haber encontrado seis variedades diferentes de maíz chococeño, es de gran importancia, por las limitaciones climáticas de la zona (altas precipitaciones, alta humedad relativa y fuerte nubosidad) para otros materiales de maíz mejorados genéticamente, las cuales tienen muchas dificultades adaptativas.

\section{CONCLUSIONES}

- El uso y el manejo que los Embera han dado al maíz chococeño, ha permitido el desarrollo de una alta diversidad intraespecífica. En el caso concreto de esta investigación hace referencia a seis variedades de maíz, lo que permite considerar sus sistemas de producción como bancos de germoplasma in situ.

- Las líneas genéticas del maíz chococeño reconocidas y descritas en la presente investigación muestran un alto potencial germinativo y de viabilidad superior a $90 \%$ en la mayoría de los casos, que comprende un período de almacenamiento entre 3 y 9 meses mostrando una alta adaptabilidad, vigor y potencial germinativo a nivel de post-cosecha, lo cual es de gran importancia para estas comunidades, donde no existen muchas técnicas de conservación de las semillas.

El cultivo de maíz en las comunidades Embera está altamente integrado en el ecosistema donde se desarrolla, conviviendo con un sin número de otras plantas que se constituyen en muchos casos en hospederos de entomofauna benéfica.

La preservación de la biodiversidad de maíz encontrada en las comunidades Embera del Atrato medio, está relacionada con el conocimiento indígena, lo que se refleja en los distintos usos que se le da a dichas variedades, las cuales están altamente vinculadas con la dieta alimenticia de esta población. Por tanto, trabajar por la seguridad alimentaria implica usar y fortalecer el conocimiento y los sistemas de producción indígenas como elemento central.

El potencial de estas variedades hace necesario que sean tenidas en cuenta en los proyectos de seguridad alimentaria en la región del Atrato medio, porque responden positivamente a las condiciones ambientales que predominan en dicha zona, expresada en altas precipitaciones, humedad y nubosidadc que tiende incluso a que se comporten agronómicamente mejor que otras variedades mejoradas, como lo han mostrado algunos trabajos realizados en el Pacífico Colombiano como el de Márquez (1994).

Las variedades de maíz chococeño, descritas aquí son altamente productivas en términos de los insumos y de la energía utilizada por estas comunidades, pues no dependen para su producción de agroinsumos e involucra muy poco trabajo, porque no se realizan desyerbes, aporques ni aplicación de insumos, por lo cual el incentivo a su siembra constituye, además de una propuesta de cultivo alimenticio, un potencial en la gestión ambiental.

Si bien este artículo se acerca a conocer algunos aspectos del vigor y la calidad de las semillas de las variedades de maíz presentes en el Atrato medio, es importante realizar más pruebas especialmente a nivel de campo. 


\section{Bioetnia Volumen 5 № 1 (enero-junio), 2008}

\section{RECOMENDACIONES}

- Es importante que se pueda hacer una caracterización, evaluación y comportamiento agronómico en campo de las variedades de la raza chococeño presentes en la zona, para poder conocer muchos más aspectos de su potencial $\mathrm{y}$ adaptabilidad frente a otras variedades en condiciones ambientales típicas de bosque muy húmedo tropical como es el caso del Atrato medio.

- Es conveniente que se fomente el intercambio de semillas locales de las diferentes variedades del maíz chococeño entre las respectivas comunidades indígenas de la zona, para seguir afianzando el proceso de autonomía alimentaria, así como la preservación, uso y conocimiento del germoplasma de maíz que se tiene.

\section{AGRADECIMIENTOS}

Los autores de este artículo agradecen a los Cabildos Mayores de Vigía del Fuerte y Murindó, así como a las comunidades indígenas del Atrato medio, en especial a Clímaco Dumazá en el acompañamiento y amistad compartida.

\section{LITERATURA CITADA}

Arango, JU., y Peñarete, D. 2000. Estrategias de producción, extracción y protección en los territorios de las comunidades Embera de Jarapetó, Jengadó y Ñarangué (Atrato medio antioqueño). Tesis Ingeniería Agronómica e Ingeniería Forestal. Medellín: Universidad Nacional de Colombia. 235 pp.

Arango, JU. y Zuluaga, G. 2007. Seguridad alimentaria desde una perspectiva de gestión ambiental en territorios de comunida des Embera del Atrato medio antioqueño. La Paz: Fondo Indígena. $225 \mathrm{pp}$.

Betancurt, C. y Zuluaga, G. 1988. Aspectos agrotecnológicos de la comunidad Embera en las veredas Chuscas y Tuguridó, Dabeiba. Tesis Ingeniería Agronómica. Medellín: Universidad Nacional de Colombia. 106 pp.

Castro, C. y Cortés, J. 1992. Comportamiento de tres variedades de maiz (Zea mayz) con diferentes niveles de fertilización en el municipio Olaya Herrera (Nariño). Tesis Tecnología Forestal. Medellín: Universidad Nacional de Colombia. 112 pp.

Centro Agronómico Tropical de Investigación y Enseñanza (CATIE) y Organización para Estudios Tropicales (OTS). 1986. Sistemas agroforestales. Principios y aplicaciones en los trópicos. San José de Costa Rica. 818 pp.

Gálvez, A. 1997. El binomio maíz-plátano: Alimentación y símbolos en la cultura Embera. Bol Antropol 11 (27): 55-75.

García, M. y Velásquez, V. 1992. Caracterización del sistema agrario y de recolección en las comunidades indígenas que habitan los ríos Torriquitadó y Chajeradó (Murindó, Chocó Antioqueño). Tesis Ingeniería Agronómica. Medellín: Universidad Nacional de Colombia. 247 pp.

Holdridge, L. R. 1982. Ecología basada en zonas de vida. San José de Costa Rica: Instituto Interamericano de Ciencias Agrícolas. $50 \mathrm{pp}$

Márquez, M. 1994. Evaluación agronómica preliminar del maíz chococeño, puya y variedades del ICA, bajo dos sistemas de manejo, en el municipio Olaya Herrera (Nariño). Vol 96. En Mesa, B. D. Historia natural del maíz. Medellín: Colección de autores Antioqueños. $396 \mathrm{pp}$.

Mesa, B. D. 1995. Historia natural del maíz. Medellín. CAA (Colección de Autores Antioqueños). Vol 96. 396 p.

Montoya, LM. 1993. Manual práctico de propagación de plantas. Medellín: Centro de publicaciones, Universidad Nacional. $103 \mathrm{pp}$.

Patiño, V. 1964. Plantas cultivadas y animales domésticos de América Equinoccial. Tomo II. Plantas alimenticias. Cali: Imprenta Departamental. $364 \mathrm{pp}$. 